\title{
Factores de riesgo asociados a la sepsis neonatal en el hospital Nuevo Amanecer
}

\author{
Risk factors associated with neonatal sepsis at Nuevo Amanecer Hospital
}

\author{
Dionicio Luis Ocampo Willis ${ }^{1}$ \\ Roland Alexander charle ${ }^{2}$ \\ Teisey Teresa Allen ${ }^{3}$
}

\section{Resumen}

A fin de contribuir al fortalecimiento de la atención integral a los recién nacidos (Neonatos), se realizó este estudio titulado: Factores de riesgos asociados a las sepsis neonatales en el hospital Nuevo Amanecer, durante enero-diciembre 2013.

El estudio fue cuali-cuantitaivo, retrospectivo de corte transversal, con resultados más destacados, siendo la edad materna con $66 \%$ entre edades de $20-34$ años, según la etnia predominan las madres miskitus en un $68 \%$, en el estado civil madres de unión libre fueron mayoritarias con $42 \%$, en cuanto a los factores maternos se destaca como IVU (Infecciones de Vías Urinarias) con 31\%, madres que cursaron con pre-eclampsia con $22 \%$, así mismo los factores de riesgo más frecuentes como bajo peso al nacer con $45 \%$, y seguido de prematuro de $29 \%$, en cuanto a los factores de riesgo relacionadas al parto no son relevantes en nuestro medio ya que en la sala de labor y parto se cumple con la normativa de asepsia y antisepsia, y se realiza buena técnica de lavado de la región vulvo perineal, previo a la atención del parto, tomando en cuenta estos resultados se realizó recomendaciones a diferentes niveles como el Hospital Nuevo Amanecer, SILAIS y también a las usuarias de los diferentes servicios de salud.

Palabras clave: Sepsis neonatal; factores de riesgo; factores de riesgo prenatales; prematura.

\section{Abstract}

In order to contribute to the strengthening of comprehensive care for newborns (Neonates), this study entitled: Risk factors associated with neonatal sepsis was carried out at Nuevo Amanecer Hospital, during January-December, 2013.

The study was qualitative-quantitative, retrospective with a cross-section scope, with more outstanding results, with maternal age being $66 \%$ between the ages of $20-34$ years, depending on the ethnic group, Miskitus mothers predominate in $68 \%$, in marital status mothers of Free union were the majority with $42 \%$, in terms of maternal factors it stands out as UTI (Urinary Tract Infections) with 31\%, mothers who had pre-eclampsia with $22 \%$, as well as the most frequent risk factors such as underweight at birth with $45 \%$, and followed by $29 \%$ premature, regarding the risk factors related to childbirth are not relevant in our environment since in the labor and delivery room the regulations of asepsis and antisepsis are complied with, and Good washing technique of the perineal vulvo region is performed, prior to delivery care, taking into account these results, recommendations were made at different levels such as Nuevo Amanecer Hospital, SILAIS and also to the users of the different health services.

Keywords: Neonatal sepsis; risk factors; prenatal risk factors; premature.

\footnotetext{
1 Estudiante de Medicina Intercultural URACCAN recinto Bilwi, E-mail: dionicioluisocampo@gmail.com, 0000-0002-7787-9178

2 Estudiante de Medicina Intercultural URACCAN recinto Bilwi, E-mail: pdiolvin@yahoo.com, 0000-0001-5165-1637

3 Licenciada en Psicología, docente de URACCAN recinto Bilwi, E-mail: tereallen.bilwi@uraccan.edu.ni, 0000-0002-8404-6318
} 


\section{Introducción}

Este estudio valora los factores de riesgo asociados a sepsis neonatal en el hospital Nuevo Amanecer, que presentaron los recién nacidos ingresados en este hospital durante el período de enero-diciembre 2013.

Las infecciones del feto y el recién nacido son una causa importante de morbilidad, mortalidad y secuelas en el recién nacido (RN). Las características propias de la etapa fetal hacen que las infecciones que ocurre en este período tengan una patogenia especial y produzcan una infección con características únicas. Estas varían según el semestre del embarazo que ocurren. En el período neonatal las características propias de la inmunidad del RN le dan también una forma de presentación y evolución característica.

Se entiende por sepsis neonatal aquella situación clínica derivada de la invasión y proliferación de bacterias, hongos y virus en el torrente sanguíneo del RN (recién nacido), y que se manifiesta dentro de las primeras 28 días de vida, si bien actualmente se tiende a incluir las sepsis diagnosticadas después de esta edad, en recién nacido con muy bajo peso al nacer (RNMBP). Es de conocimiento que el 30-40\% de las muertes neonatales se encuentran asociadas a infecciones. De cada 100 muertes infantiles (<1año), 71 son neonatos y entre un 10-50\% de las muertes neonatales hospitalarias son debidas a la septicemia neonatal (MINSA,2013).

En Nicaragua la mortalidad neonatal en los últimos tres años ha representado más del 60\% de las muertes infantiles, más del 7\% ocurrió en etapas tempranas. Las sepsis neonatales precoz generalmente se presenta como una enfermedad fulminante y multisistémica más frecuente durante los primeros 3 días de vida. Estos recién nacidos han tenido historia de uno o más factores de riesgo (prenatales, la inadecuada atención del parto y del recién nacido), y/o enfermedades nosocomiales adquiridas en unidades hospitalarias por procedimientos invasivos y en otros casos puede ocurrir por infecciones adquiridas en la comunidad (parto domiciliar o egresos tempranos.

Por lo tanto, consideramos que los resultados de esta investigación son de mucho valor para nosotros como investigadores, trabajadores de la salud (médicos, enfermeras, estudiantes de medicina), también para el sistema de salud de la región en tomar acciones de promoción, prevención, curación y rehabilitación con el fin de mejorar los servicios de salud de atención de este grupo etario teniendo en cuenta que son de mucha prioridad para el Ministerio de Salud y para el desarrollo como país y región, específicamente en pro de la erradicación de esta problemática, ya que es uno de los indicadores de la eficacia de la atención que se brinda a este grupo de pacientes.

\section{Revisión de literatura}

\section{Principales factores en el riesgo de sepsis neonatal}

Fernández y col. (2013) expone en su investigación sobre la Sepsis Neonatal que: Es una infección bacteriana con invasión al torrente sanguíneo del recién nacido, con respuesta inflamatoria inespecífica y manifestaciones clínicas atípicas, adquirida de la madre en forma ascendente o transplacentaria, y debido a factores de riesgo maternos. La nomenclatura para su identificación según CIE-10 está comprendida en las infecciones específicas del período perinatal (p.85 89).

Gómez, F. (2011), afirma: "Las sepsis de origen bacteriano constituye una de las principales causas de morbi-mortalidad en el período neonatal". La sepsis es causada por microorganismos que han variado a través del tiempo debido a estrategias globales de prevención, por una parte y por otra, a las características propias de los recién nacidos $(\mathrm{RN})$ hospitalizados, procedimientos y tratamientos que ellos reciben. Dado que los mecanismos de inmunidad de los recién nacidos son relativamente deficientes en comparación con lactantes y niños mayores, siendo esto de especial relevancia en prematuros y de bajo peso de nacimiento en los cuales, la inmadurez del sistema inmune se acompaña de otros factores de riesgo y de procedimientos invasores que aumentan la vulnerabilidad a infecciones. 


\section{Factores neonatales que inciden en el riesgo de sepsis neonatal}

El factor de riesgo neonatal más importante es la prematurez; se ha descrito incidencia en sepsis 8 a 10 veces mayor, si comparamos niños de 100 a 1500 gramos en relación al grupo de 200 a 2500 gramos y la tasa para sepsis por Streptococo grupo B fue 26 veces mayor para menores de 1000 gramos en comparados con mayores de 2500 gramos (Gómez, F.,2011).

Se ha descrito el riesgo de desarrollar sepsis se debe en parte a la mayor vulnerabilidad de las barreras naturales y en parte al compromiso del sistema inmune del neonato, como, por ejemplo, la trasferencia placentaria materna de IgG al feto recién comienza a las 32 semanas de gestación e IgA secretora está muy disminuida tanto en los pulmones como en el sistema gastrointestinal. Además, las barreras físicas naturales son inmaduras, especialmente piel, cordón umbilical, pulmón e intestino, así mismo hay una disminución de la actividad de la vía alterna del complemento $\left(C_{3}\right)$, lo cual conlleva a una diferencia en la opsonización de los gérmenes con cápsula polisacárido. (Fernández, Colomer, y Cotallo, 2013).

Por otra parte, el rápido agotamiento de los depósitos de los neutrófilos maduros medulares cuando hay exposición a una infección ya que estos neutrófilos tienen menor capacidad de adherencia y fagocitosis y menor capacidad bactericida. La inmunidad mediada por linfocito T helper y linfocito NK (natural killer) está alterada y la memoria inmunológica es deficiente por lo que a mayor prematuridad hay más inmadurez inmunológica y mayor frecuencia de infecciones.

Algunas condiciones que se consideran como factor de riesgo, como la ventilación mecánica, alimentación parenteral, cateterismo, etc.; los usuales si no se realizan sobre medidas estrictas de asepsia y antisepsia puede colonizar el torrente sanguíneo del neonato por microorganismos predominantes en la piel los cuales son patógenos capaces de desarrollar sepsis.

\section{Factores maternos que inciden en el riego de sepsis neonatal}

los factores obstétricos más importantes incluyen: trabajo de parto prematuro, rotura prematura ovular (RPO) de más de 18 horas, colonización materna con gérmenes específicos, corioamnionitis y fiebre materna en el parto. Así para madres colonizadas con estreptocos grupo B la incidencia se septicemia precoz es de 1-2\% pero esta sube a 15\% si hay trabajo de parto prematuro o a $10 \%$ si hay corioamnionitis o RPO de más de 24 horas; si asociamos factores de riesgo la probabilidad d septicemia sube aún más, pudiendo ser de un $25 \%$ a un $30 \%$ si hay portación materna de estreptococo B además de corioamnionitis. La mortalidad en la forma tardía es de $2-6 \%$ y la prematurez es infrecuente. Para la forma precoz sin embargo la prematuros en un $30 \%$ de los casos y la mortalidad es de $10 \%$ en RN de término y de $25-30 \%$ en prematuros". (Carlos Osorio, C.A y Vásquez, A.R., 2005).

Según Alvarenga, B. (2003). Los factores de riesgos prenatales que influyeron en la presencia de sepsis en el recién nacido. La infección de vías urinarias y las servicovaginitis tanto durante en el embarazo como el parto con valores muy estadísticos muy significativos. Otros factores de muy importancia lo constituyo el control prenatal y la manipulación previas a su ingreso hospitalario. Se comparó los controles prenatales entre casos $(6.8 \%)$ y controles $(16.8 \%)$ con un valor estadístico p o.11, $\mathrm{OR}=0.36$, las mujeres que se realizaron menos $4 \mathrm{CPN}$ no se encontró diferencia entre los casos y sus controles.

Consideramos citar los factores de riesgos maternos y clasificarlo:

\section{Factores de riesgos mayores}

1. Ruptura prematura o prolongada> de $24 \mathrm{~h}$.

2. Fiebre materna intraparto $>38 \mathrm{c}$ ).

3. Corioamnionitis (T materna $>38 \mathrm{c}, \mathrm{FCF}>160$ latidos por minuto. Sostenida, dolor uterino, taquicardia materna, secreción vaginal fétida, $G B>20,000$ ). 
4. Infección de vías urinarias no tratadas.

5. Trabajo de parto pre termino.

6. Pre eclampsia.

\section{Factores de riesgos menores}

1. Ruptura prematura (antes del TP) o prolongada (con TP activo) de membranas (> de 12h.).

2. Fiebre materna intraparto (> 37.5c).

3. Glóbulos blancos maternos $>15,000 / \mathrm{m}$.

4. Loquios fétidos.

5. Edad materna.

Este tipo de estudios, permite cuantificar los grupos de riesgo, o sea aquellos grupos que tengan mayor posibilidad de daño (alto riesgo) o menor (bajo riesgo). Este enfoque basado en el concepto de riesgo, tiene gran trascendencia desde el punto de vista sanitario y con miras a encararlo de esta forma, se adopta en el estudio mencionado la clasificación propuesta por Buttler y Bonham en 1958 y utilizada con éxito en numerosos estudios ulteriores.

Las madres son categorizadas en tres niveles de riesgo en relaciona con su edad:

1. Alto riesgo: menos de 20 años.

2. Bajo riesgo: entre 20-34 años.

3. Riesgo intermedio: mayores de 34 años.

Desde el punto de vista de la atención materno infantil se define como riesgo a la mayor probabilidad de producirse daño (muerte, enfermedad, y/o secuela) en el periodo de referencia (en este caso el primer año de vida.

La edad materna es un factor que se asocia significativamente con posibilidad de daño al hijo (mortalidad) durante el primer año de vida y permite identificar y cuantificar dónde y de que dimensión es el riesgo a que está sometido es niño.

\section{- Nivel de escolaridad}

El nivel de instrucción materna es un factor de riesgo para el hijo en cuanto a la probabilidad de sufrir un daño (muerte, enfermedad y/o secuela).

En principios y recomendaciones de las Naciones Unidas, a través de su oficina de estadística, recomienda en categorizar el nivel de instrucción materno en siete grupos: analfabeta, primaria completa e incompleta, secundaria completa e incompleta, universitaria completa e incompleta.

Es de relevancia la importancia del grado de instrucción de toda mujer gestante acerca de señales de peligro, alimentación, higiene personal, lo cual interviene de manera directa en la prevención y detección temprana de dichas patologías durante el embarazo.

El mayor riesgo corresponde a los niños, hijos de madres con bajo nivel de instrucción y el menor riesgo a los hijos de madres con alto nivel de instrucción.

La educación materna ha demostrado una relación inversamente proporcional con la morbi-mortalidad neonatal. Por lo que se concluye por algunos investigadores que, las madres cuyo nivel de instrucción es alto disponen de mejores cuidados médicos para el control del embarazo y parto, lo cual es importante para detectar señales de peligro, factores de riesgo y patologías durante el embarazo los cuales podrían desarrollar sepsis al recién nacido. 


\section{- Etnia de las madres}

En nuestro medio, como una región pluricultural, multilingüe donde hay intercambio de diversas etnias, prácticas culturales, diferentes cosmovisiones consideramos de importancia investigar en qué grupo étnico se presenta más esta problemática.

\section{- Estado civil}

Esta variable se ha asociado sobre todo a un riesgo aumentado por el bajo peso al nacer y guarda estrecha relación con otros factores de riesgo como el embarazo en adolescencia, consumo de alcohol y tabaquismo.

En un estudio sobre 36.608 nacimientos en el distrito de Columbia, se encontró un 34\% más de incidencia de bajo peso y $35 \%$ más de mortalidad neonatal en niños nacidos de madres solteras, el riesgo permaneció importante a pesar del control por edad y educación materna.

En un análisis de la mortalidad infantil y neonatal en Chile de 1965 a 1990, observaron que la ilegitimidad incrementó de 17.45 a 34.3\% constituyéndose en uno de los principales problemas médico-sociales que trascienden en mortalidad infantil y neonatal a pesar de haber mejorado aspectos como la educación materna.

\section{Factores de riesgo en el recién nacido}

El factor de riesgo neonatal más importante es la prematurez, se ha descrito incidencia de sepsis 8 a 10 veces mayor si comparamos niños de 1000 a 1500 gramos en relación al grupo de 2000 gr a 2500 gr y la tasa para sepsis por Streptococo grupo B fue 26 veces mayor para menores de 1000 gramos comparados con mayores de 2500 gr. Federico Gómez (2011).

Algunas condiciones que se consideran como factor de riesgo, como la ventilación mecánica, alimentación parenteral, cateterismo, etc.; los usuarios si no se realizan medidas estrictas de asepsia y antisepsia puede colonizar el torrente sanguíneo del neonato por microorganismos predominantes en la piel los cuales son patógenos y capaz de desarrollar sepsis.

\section{Factores de riesgo asociados al parto que conllevan a sepsis neonatal}

El parto es el conjunto de fenómenos fisiológicos que determinan y acompañan la expulsión del feto y los anexos ovulares, desde la cavidad uterina a través del canal del parto.

La aplicación igualitaria de estos adelantos no ha sido posible en todos los lugares del mundo, más propiamente en los países subdesarrollados donde existe una alta tasa de atención domiciliaria de los partos. Estos conllevan a altos niveles de mortalidad debido principalmente a complicaciones en la segunda etapa del parto, atención no especializada del recién nacido de alto riesgo.

Existen diversos factores de riesgos para sepsis neonatal los cuales están íntimamente ligados al parto como lo son el traumatismo mecánico durante el parto, los cuales provocan soluciones de continuidad dejando como puerta de entrada a los microorganismos patógenos y así colonizar el torrente lo cual posteriormente desarrolla sepsis neonatal.

Por otra parte, el parto domiciliar se considera como factor de riesgo para el desarrollo de sepsis neonatal sabiendo que la atención al parto y principalmente al neonato no se realiza en condiciones óptimas, refiriéndose a las técnicas de asepsia y antisepsia, y más si a esto se la mala higiene de la región perineovulvar predispone más aun al neonato a contaminarse con los gérmenes que se encuentra en el canal del parto. 


\section{Material y métodos}

\section{Tipo de estudio}

Cuali-cuantitativo, retrospectivo de corte transversal, realizado en el período comprendido enero-diciembre 2013.

Área de estudio

Servicio de Neonatología del Hospital Nuevo Amanecer "Nancy Bach".

\section{Unidad de análisis}

Todo neonato ingresado en neonatología, durante el período de estudio.

\section{Universo}

44 casos diagnosticados con sepsis neonatal en el período de estudio.

\section{Muestra}

44 expedientes clínicos.

\section{Tipo de muestra}

Muestreo no probabilístico.

\section{Fuentes y formas de obtención de la información}

\section{Primarias:}

Las fuentes primarias fueron los expedientes clínicos, libro de registros mensual del servicio neonatológico y registro de base de datos del Hospital Nuevo Amanecer.

Observación en sala de alto riesgo obstétrico, labor y parto sobre la atención al parto. Así también las entrevistas a madres de recién nacidos.

\section{Secundarias}

Revisión bibliográfica, webgrafías, internet, artículos.

\section{Técnicas e Instrumentos}

Para la recolección de los datos se elaboraron instrumentos fichas y guías de encuestas, entrevistas que responde a los objetivos, el cual se utilizó para la obtención de información útil en proceso investigativo.

\section{Criterios de inclusión:}

- Neonatos.

- Expedientes clínicos completos neonatales y maternos.

- Ingresados durante el periodo de enero a diciembre 2013. 
- Expedientes con diagnóstico de sepsis neonatal.

\section{Criterios de exclusión:}

- Expedientes incompletos.

- Expedientes con diagnósticos presuntivos de sepsis neonatal 2013.

\section{Consideraciones éticas}

Mecanismos de procesamiento y análisis de datos durante la fase de organización fue coordinada con responsables inmediatos para la adquisición de información, además la autorización de la directora del Hospital Nuevo Amanecer (HNA), para la revisión de los registros de cada expediente de pacientes incluidos como elemento en la población de estudio, para lo cual se le extendió una carta solicitando su aprobación.

Se realizó entrevista directa a domicilio a 13 madres de los neonatos incluidos en el estudio, a las cuales se les explicó el motivo y propósito de la entrevista que es de carácter voluntario, se realizó la entrevista con previo consentimiento de las encuestadas.

La identidad de las encuestas fue anónima para guardar sus identidades. Una vez obtenidos la información se procedió registrar en nuestro instrumento. Posteriormente, analizados en base a datos creados en Microsoft Excel, 2010. Los resultados se presentan en tablas y gráficos.

\section{Resultados y discusión}

Los resultados que a continuación se detallan fueron descritos a partir de los datos estadísticos del servicio de neonatología del Hospital Nuevo Amanecer e información recolectada a través de las encuestas y entrevistas a las madres que tenían hijos ingresados al servicio de neonatología incluido en el periodo de estudio. La información se describe de acuerdo al orden cronológico de los objetivos específicos de la investigación.

\section{Características sociodemográficas}

\section{- Edad maternal}

Teóricamente autores como Buttler y Bonham (1958), clasifican las edades de riesgo como alto riesgo menos de 20 años, bajo riesgo entre 20-34 años y riesgo intermedio mayores de 34 años. Sin embargo, en nuestra investigación como resultado encontramos los siguientes hallazgos, los cuales no concuerdan con la teoría, consideramos que en nuestro medio la edad de mujer potencialmente fértil se encuentra entre mujeres de 20-34 años también. Por otro lado, los hallazgos de madres dentro del grupo etario considerado de alto riesgo obstétrico que comprende edades mayores de 35 años con $23 \%$ donde es más frecuentes los embarazos de alto riesgo, y algunas patologías neonatales frecuentes relacionadas como bajo peso al nacer, malformaciones congénitas que sí guardan relación con el desarrollo de sepsis neonatal.

De los 44 casos de sepsis neonatal que se encontraron en las edades de las madres de neonatos con sepsis neonatal, predomina en las edades de $20-34$ años de edad en un $66 \%$, posteriormente en madres mayor de 35 años con $23 \%$, y luego madres menores de 20 años en un $11 \%$. 
El gráfico adjunto presenta los resultados de las edades maternas las cuales tuvieron hijos con sepsis neonatal.

\section{Edades maternas}

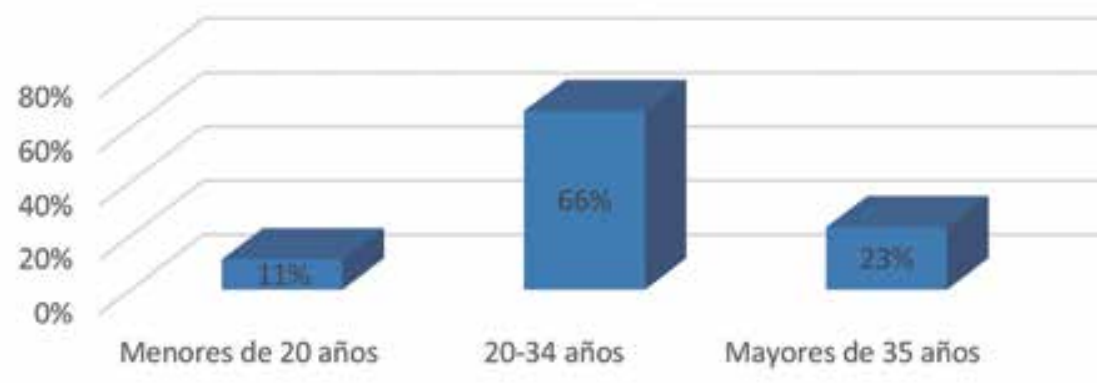

\section{$\checkmark$ Etnia}

En cuanto a la etnia predominante de las madres se encontró la etnia miskitu en un $68 \%$, y la etnia mestiza en un $32 \%$, no se encontraron madres de etnia mayagna ni de la etnia creole.

El gráfico adjunto presenta los resultados según la etnia más afectada con sepsis neonatal.

\section{Etnia de las madres}

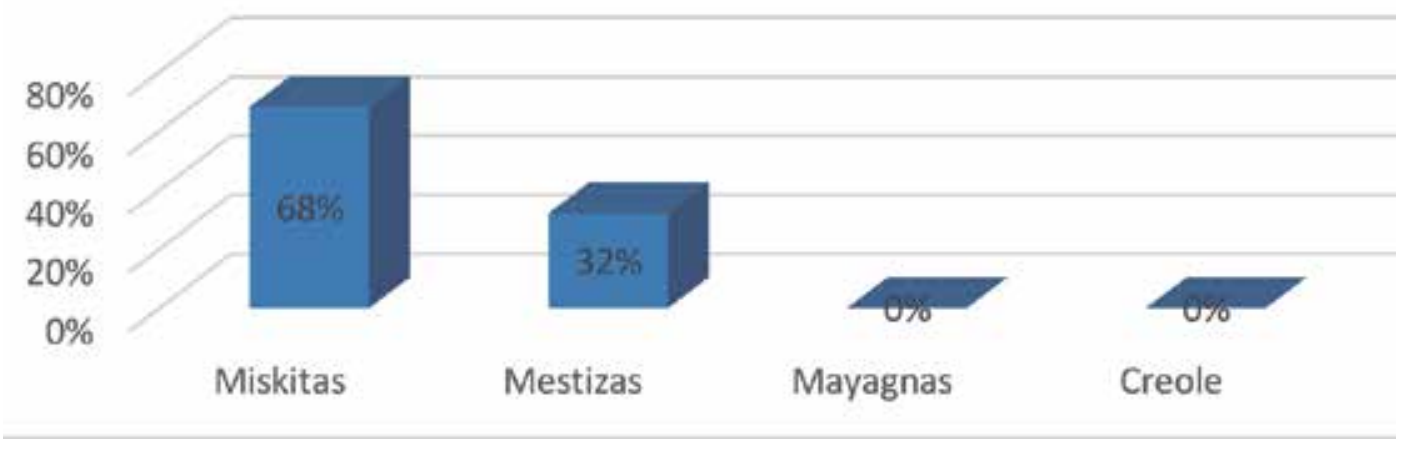

Dicho hallazgo se justifica con las particularidades sociodemográficas de la región donde se sabe que la población mayoritaria en la región pertenece a la etnia mestiza; sin embargo, en nuestro municipio Puerto Cabezas la población miskitu es la mayoritaria, lo cual concuerda con la teoría del autor Yuri Zapata y su estudio sobre historiografía de la Costa Caribe. Cabe destacar que para atenciones de salud como usuarias, la población mayoritaria es de la etnia miskitu. 


\section{- Estado Civil}

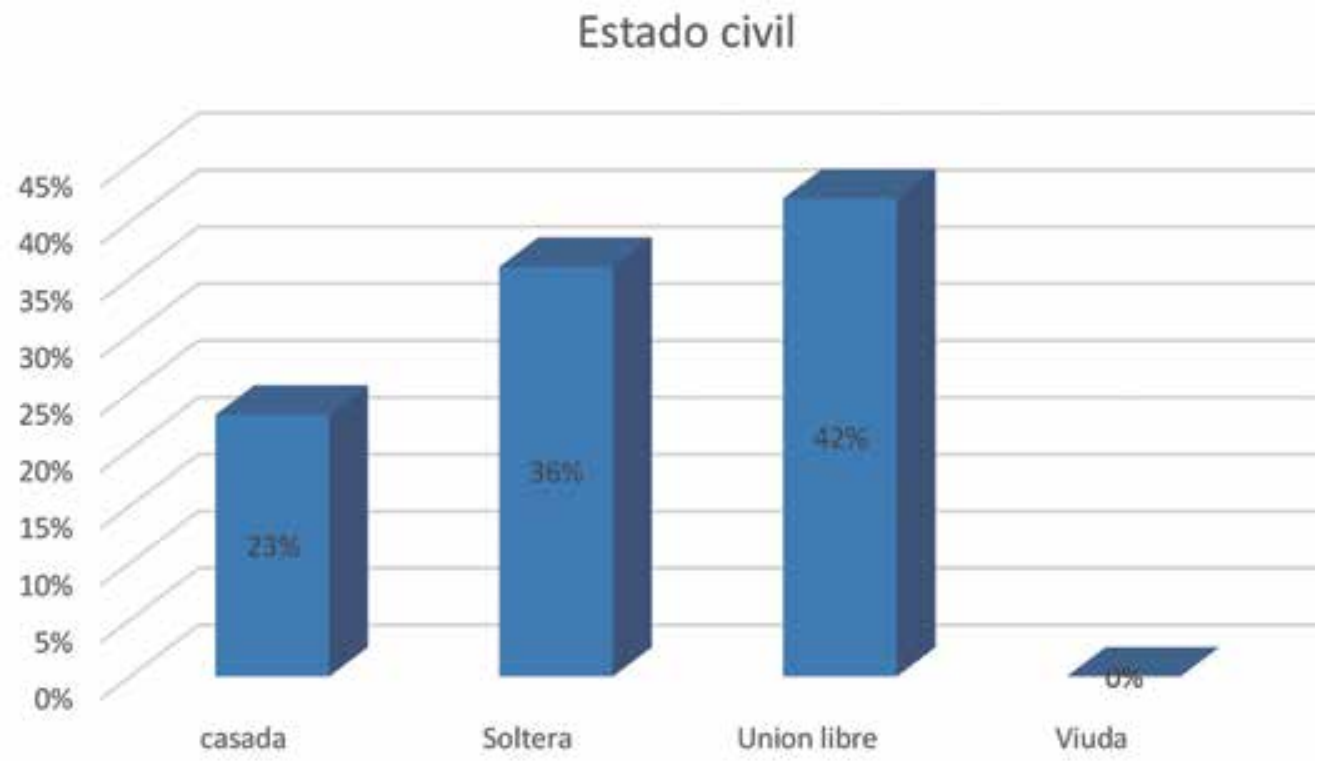

Por otro lado, el estado civil de las madres de neonatos con sepsis neonatal se encontró en un $42 \%$ que viven en unión libre, en un $35 \%$ que son solteras, y en un $23 \%$ que son madres casadas.

Mayoritariamente en la unión libre, consideramos las responsabilidades de los progenitores para la preparación de recibir un recién nacido, la legalidad de la parte familiar tanto los compromisos existentes en la pareja. Además, dicho hallazgo guarda estrecha relación con la educación paterna y materna sobre el cuido de los recién nacidos en cuanto al aseo del neonato, alimentación adecuada de acuerdo a la edad.

\section{- Procedencia}

Según la procedencia de estas madres se determinó que en un $68 \%$ son madres procedentes del área urbana y un 32\% madres procedentes del sector rural. La procedencia de las madres que predominan son las del área urbana, se piensa que es por lo mismo que el estudio fue realizado en este medio y el hospital que está ubicado en la zona urbana, de tal manera son más frecuentes las mujeres de la zona urbanas las que son más atendidas.

\section{Procedencia de las madres}

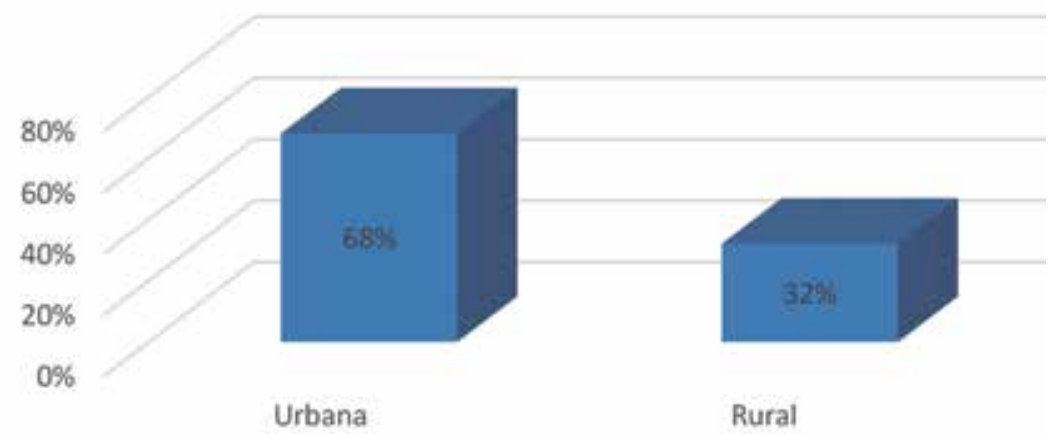




\section{- Nivel de escolaridad}

Según la escolaridad de las madres se determina con más frecuencia que sólo han cursado la primaria en un $41 \%$, y madres con secundaria cursada en un $34 \%$ y analfabetas con $18 \%$ y universitarias en un $7 \%$. Este hallazgo es de mucha relevancia, ya que una buena educación materna es de vital importancia, como se menciona en los párrafos anteriores que una educación materna es sinónimo de buena atención y cuido al recién nacido y por ende un mejor estilo de vida. En cada atención en sus controles prenatales el personal de salud les orienta el conjunto de herramientas para una cautela integral sobre los riesgos.

\section{Grado de escolaridad de las madres}

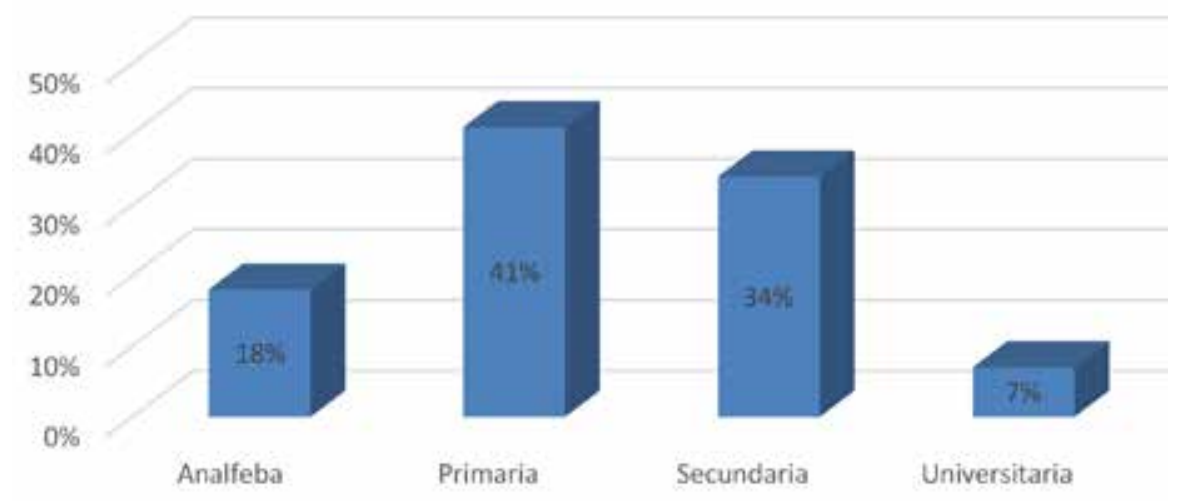

\section{- Cultura sanitaria}

Durante las visitas domiciliares se lleva a cabo las vivencias familiares, la parte del ambiente en que viven, el patio, la casa, su infraestructura y otros componentes. Los parámetros medidos fueron "bueno" o "malos" se logró identificar con un $64 \%$ como malas y un $36 \%$ buenas.

Chávez(2003) afirma que, la cultura sanitaria permite rescatar los significados de las personas alrededor de los sistemas salud -enfermedad-atención, para que puedan ser considerados al momento de estudiar o analizar dicho sistema dentro de la estructura social.

Para llevar a cabo lo anterior vamos a desarrollar en nuestro medio la cultura sanitaria, el cual tiene una dimensión específica y son los que tienen practicas deficientes higiénico sanitarias, cabe destacar que como protagonistas se tienen que mantener un ambiente limpio y tranquilo para evitar propagación de las fuentes que puede alterar el estado de salud una vez la gravedad principalmente en el período cercano al parto.

\section{Cultura sanitaria}

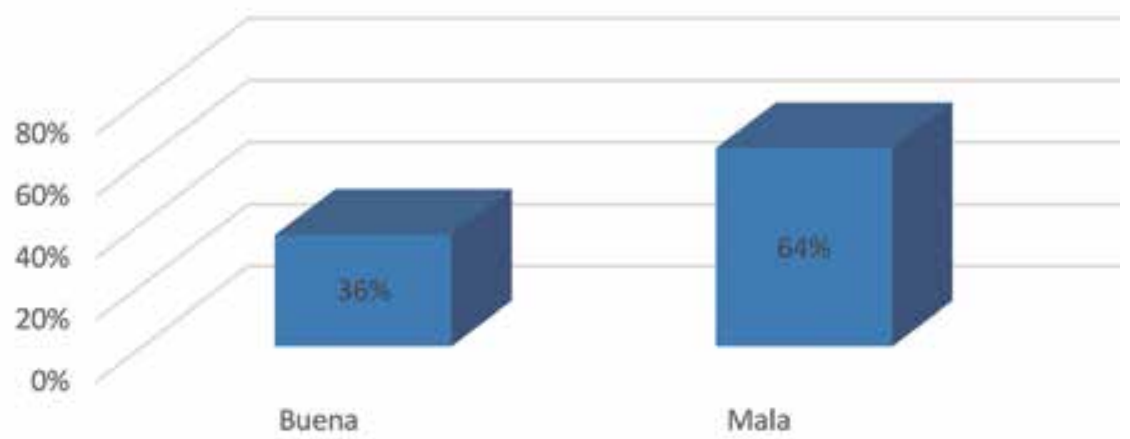




\section{$\checkmark$ Hacinamiento}

De la población estudiada un $57 \%$ se encuentra en hacinamiento y $43 \%$ que no se encuentra en hacinamiento. Aporta el autor Velazco en sus estudios en donde el hacinamiento refiere a la relación entre el número de personas en una vivienda o casa y el espacio o números de cuartos disponibles.

El hacinamiento es considerado un factor de riesgo importante para el desarrollo de muchas enfermedades, dado por la carencia de sanidad y condiciones precarias en higiene, lo cual un neonato fácilmente puede contraer infecciones y desarrollar sepsis.

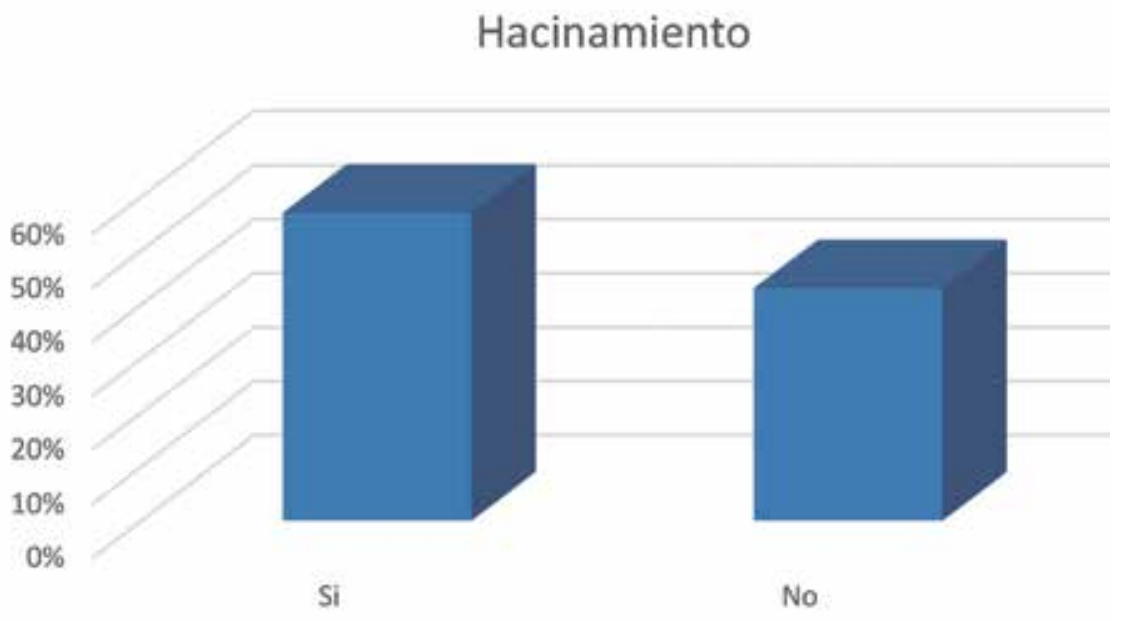

\section{- Cosmovisión de madres sobre sepsis neonatal}

\section{Cosmovision}

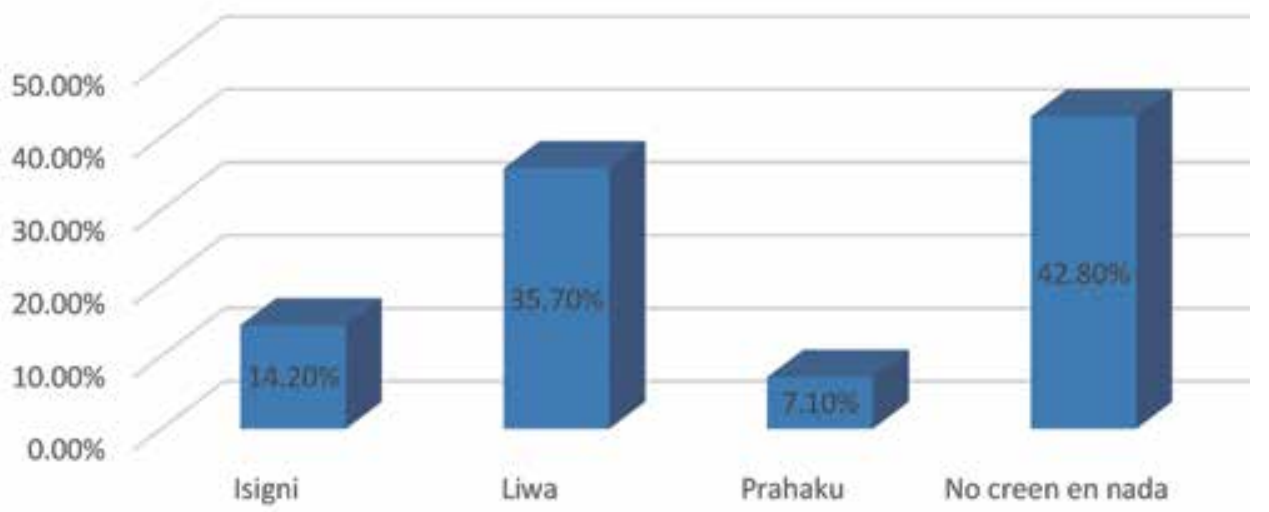

Hemos percatado que los grupos de las madres quienes han reflejado sus formas de ver el mundo desde una forma cultural desde sus raíces, los datos presentes que las que no creen son mayoritarias con un porcentaje considerable de $42.8 \%$, en cambio las que tienen credulidad a estos espíritus según sus ancestros han inculcado que el dueño del agua es responsable de afecciones cuando una vez estando embarazadas en los sueños se la perseguía y provocaba enfermedades como el flujo vaginal, además son amenazas de partos prematuros; sin embargo, una vez cuando nace el bebe es cursado y perseguido por este espíritu a través del lilka en donde el liwa lleva y lo guarda en el lugar llamado Apalka y posteriormente mata al 
neonato por tales razones se ha encontrado un porcentaje de $35.7 \%$. También han indicado las madres, que una vez fallecido el neonato se deja huella o marca de la palma de mano en el dorso del neonato del espíritu que ha provocado.

En cuanto a la cosmovisión y salud de los pueblos de nuestro medio. Según Fagoth et al. (1998), se concibe al ser humano como un ser integral, por lo que sus afectaciones también son integrales y tienen consecuencias en todo el ser. Partimos por admitir que existe una armonía ideal entre las fuerzas de la naturaleza y el wina tara (cuerpo material).

\section{- Factores de riesgo maternos}

\section{Factores de riesgo maternos}

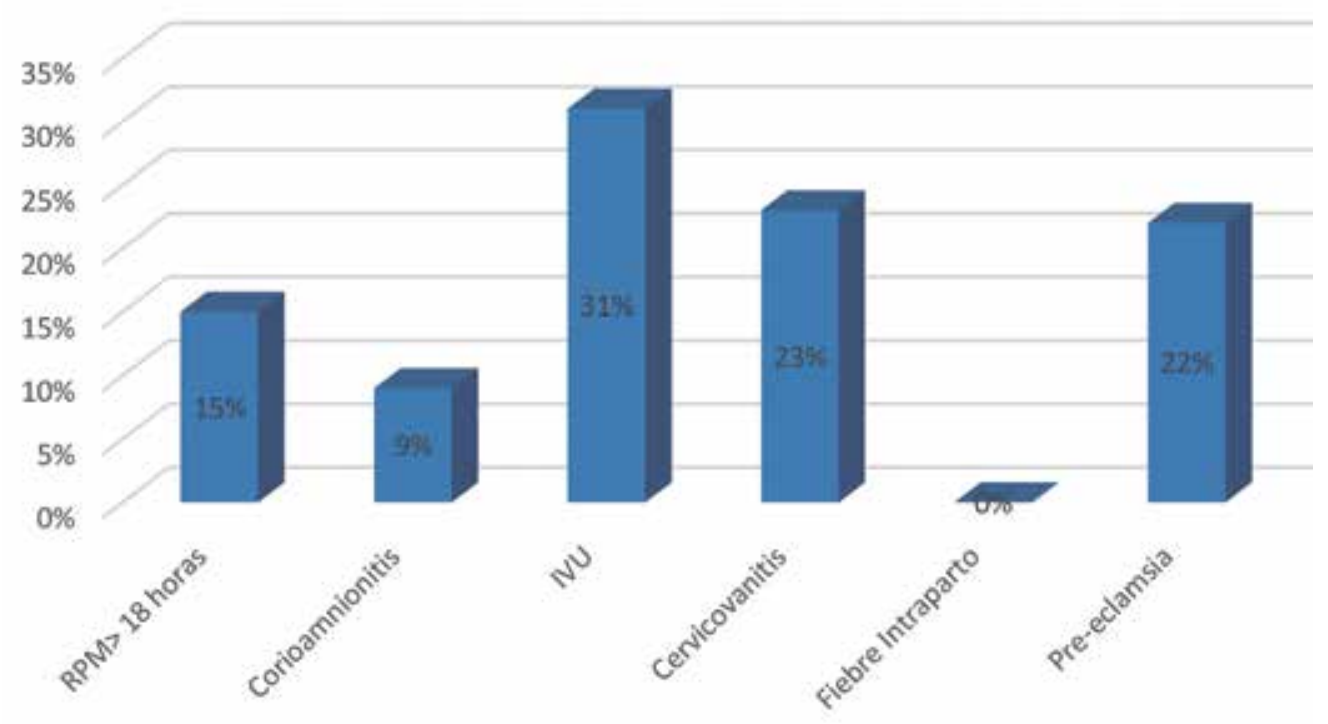

Los factores de riesgos maternos se encontraron en un alto porcentaje en las madres con infecciones de vías urinarias (IVU)infecciones de vías urinarias 31\%, así mismo madres que cursaron con pre-eclampsia con $22 \%$, siguiendo el alto porcentaje de madres con cervicovaginitis con $23 \%$, en cuanto las embarazadas que presentaron RPM mayor de 18 horas se encontraron en un $15 \%$ y corioamnionitis en un $9 \%$.

Los factores de riesgo maternos, madres con infecciones de vías urinarias no tratadas como afirman las literaturas internacionales sobre las Infección del Tracto Urinario, activa sin tratamiento y se asimila al estudio de dicho autor (Federico Gómez 2011).

Además, como agente etiológico más frecuente que causa las infecciones de vías urinarias son de origen bacteriana y el más frecuente es la E. coli (Echericha coli), que de igual manera es muy frecuente encontrar este agente infeccioso como causante de sepsis neonatal. E.coli (Echericha coli) liberan enzimas proteolíticas las cuales pueden producir lisis celular de la membrana amniótica y llegar a infectar el líquido amniótico, lo cual infecta al feto y desarrollan sepsis neonatal pueden inducir un parto prematuro. Además, los factores de riesgos maternos encontrados como madres que presentaba cervicovaginitis lo cual sospechamos de infecciones del tracto vaginal y canal del parto con el estreptococo B- hemolítico del grupo $\mathrm{B}$, gérmenes que son frecuentes en los casos de sepsis neonatal temprana. 


\section{- Factores de riesgos neonatales}

\section{Factores neonatales}

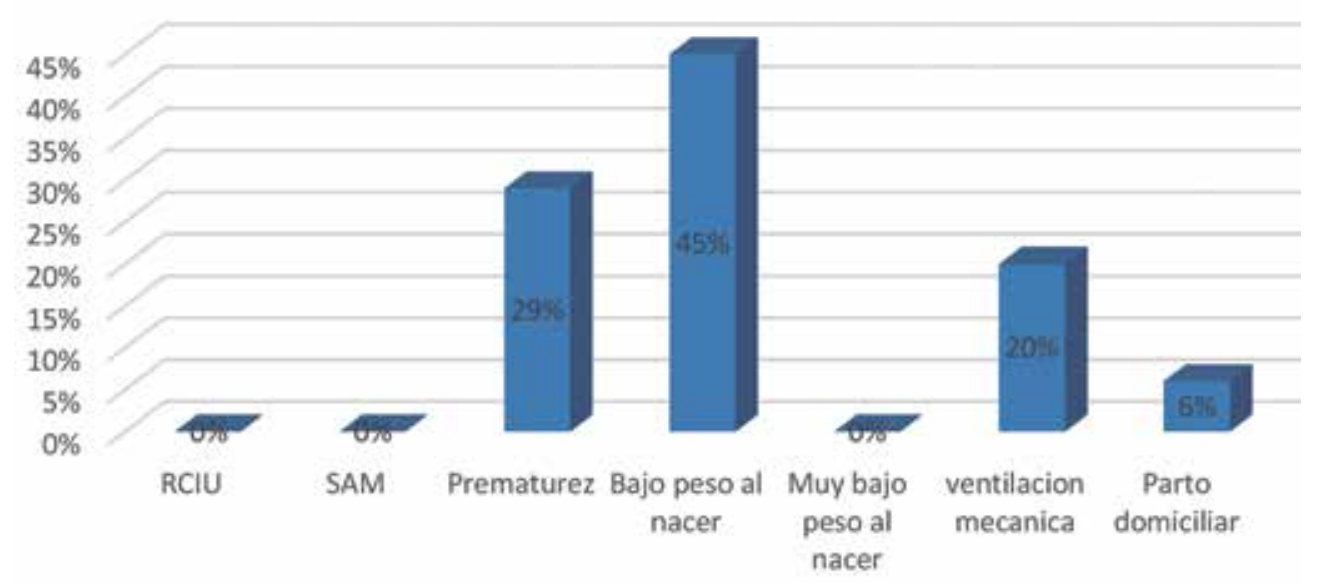

Entre los factores de riesgo neonatal en este estudio se encontró con mayor frecuencia de sepsis en neonatos con bajo peso al nacer con un $45 \%$, y posteriormente neonatos con prematurez $29 \%$, además los neonatos que recibieron ventilación asistida o mecánica desarrollaron sepsis en un $20 \%$, por otra parte, los partos domiciliares $6 \%$.

En cuanto a los factores de riesgo neonatales se destaca los neonatos con bajo peso al nacer asociado a la prematurez los cuales son muy vulnerables a desarrollar sepsis por la inmadurez de su sistema inmunológico para combatir contra agentes agresores; además, se relaciona con pacientes que fueron sometidos a ventilación mecánica, por causa de distres respiratorio, ya que estos son procedimiento invasivo lo cual si no se realiza desde una técnica de asepsia y antisepsia adecuado es considerado como un factor de riesgo potencial para desarrollar sepsis neonatal nosocomial.

\section{Conclusiones}

Tomando en cuenta los datos recabados podemos concluir que:

1. La edad de las madres con recién nacidos con sepsis neonatal predomina en un rango de edad de 20-34 años.

2. Según la etnia de las madres predomina la etnia miskitu.

3. El estado civil de las madres es más frecuente las madres que bien en unión libre

4. En cuanto la procedencia de las madres es más frecuente las madres del sector urbano.

5. Según el grado de instrucción o el grado de escolaridad prevalece más las madres con primaria cursada.

6. Las madres de los neonatos incluidos en el estudio posee una cultura sanitaria mala.

7. La población estudiada se encuentra en hacinamiento.

8. Según la cosmovisión de las madres estudiadas creen que el liwa provoca enfermedades al recién nacido. 
9. Los factores de riesgos maternos más encontrados son infecciones de vías urinarias, cervico vaginitis, y pre eclampsia, lo cual son factores de riesgos potenciales para sepsis neonatal.

10. Factores de riesgos neonatales, los casos de sepsis neonatal se ven más frecuentes en recién nacidos con bajo peso al nacer y sobre todo en pretérminos.

11. No se encontró datos sobre factores relacionados con el parto, el cual pretendía recopilar información acerca de ello.

\section{Agradecimiento}

Esta publicación obtuvo el financiamiento de: El Fondo de Asistencia Internacional de los Estudiantes y Académicos Noruegos (SAIH).

\section{Lista de referencia}

Alvarenga, B.M. Bravo, S. Suarez, F. \& Leyton A. (2003). Factores asociados a sepsis neonatal en nacimientos ocurridos en el hospital Bertha Calderón, productos de madres procedentes del municipio de Managua, en el periodo de julio a septiembre del 2003. Marzo 07, 2016 de MINSA. http://campos.easp.es/abierto/file. php?file=\%2F78\%2FInvestigacion\%2FFactores de riesgos asociados a sepsis neonatal-EDIT-2.pdf.

Buttler,N. R \& Bonhman D . G (2005). Perinatal Mortaly. Britisth Perinatal Mortaly Survey, Edimburgo, Livingston.

Fernández, Colomer. B \& Cotallo, D. (2013). Sepsis del recién nacido. Servicio de Neonatología, Hospital Universitario Central de Asturias.

Gómez, F. (2011). Sepsis bacteriana en el recién nacido. Guías clínicas del departamento de neonatología Hospital Infantil de México, México. DF. Capítulo 1, (pp.2 -8).

MINSA (2013). Guías clínicas para la Atención del Neonato, Gobierno de Reconciliación y Unidad Nacional Ministerio de Salud./. Managua: MINSA, (pp 270-276).

Osorio, A. C, \& Vásquez, R. A. (2005). Factores de riesgo asociado a mortalidad neonatal precoz. Servicio de neonatología en el hospital general (Dr. Gustavo A. Povirosa Pérez, Tabasco).

Zapata, W. Y (2007). Historiografía, Sociedad y Autonomía /Yuri Hamed Webb.1ed-Managua: URACCAN. (PP. $.17-32)$. 